\title{
Reliance on end-expiratory wedge pressure leads to misclassification of pulmonary hypertension
}

\author{
Barbara L. LeVarge1, Eugene Pomerantsev² and Richard N. Channick ${ }^{3}$ \\ Affiliations: \\ 'Dept of Pulmonary and Critical Care Medicine, Beth Israel Deaconess Medical Center, Boston, MA, USA. \\ ${ }^{2}$ Dept of Cardiology, Massachusetts General Hospital, Boston, MA, USA. \\ ${ }^{3}$ Dept of Pulmonary and Critical Care Medicine, Massachusetts General Hospital, Boston, MA, USA.
}

Correspondence: Barbara L. LeVarge, Dept of Pulmonary and Critical Care Medicine, Beth Israel Deaconess Medical Center, 330 Brookline Avenue, Boston, MA 02215, USA.

E-mail: blevargeabidmc.harvard.edu

ABSTRACT Current guidelines recommend measurement of pulmonary artery wedge pressure (PAWP) at end-expiration. However, this recommendation is not universally followed and may not be physiologically appropriate. We investigated the performance of end-expiratory PAWP in the evaluation of precapillary pulmonary hypertension patients.

329 spontaneously breathing patients undergoing right heart catheterisation were retrospectively classified as having a precapillary, post-capillary or mixed phenotype based on standardised clinical criteria. Tracings were reviewed to compare end-expiratory PAWP with PAWP averaged throughout the respiratory cycle; these values were correlated with the clinical classifications. Predictors of large respirophasic variation in PAWP were determined.

Elevated end-expiratory PAWP (>15 mmHg) occurred in $29 \%$ of subjects with precapillary phenotype. There were no differences in demographics or clinical history between those with elevated and normal endexpiratory PAWP. Those with elevated end-expiratory PAWP had greater right atrial pressure and respirophasic PAWP variation. Among all subjects, the magnitude of respirophasic variation in PAWP was positively correlated with body mass index and respirophasic variation in left ventricular end-diastolic pressure.

A significant proportion of precapillary pulmonary hypertension patients have end-expiratory PAWP $>15 \mathrm{mmHg}$. Spontaneous positive end-expiratory intrathoracic pressure may contribute; in those cases, PAWP averaged throughout respiration may be a more accurate measurement.

@ERSpublications

Strict use of end-expiratory pulmonary artery wedge pressure leads to over diagnosis of pulmonary venous hypertension http://ow.ly/vNHMj

Received: Nov 302013 | Accepted after revision: April 032014 | First published online: June 122014

Support statement: This study was conducted with support from Harvard Catalyst, the Harvard Clinical and Translational Science Center (National Center for Research Resources and the National Center for Advancing Translational Sciences, National Institute of Health Award UL1 TR001102 and financial contributions from Harvard University and its affiliated academic healthcare centres). The content is solely the responsibility of the authors and does not necessarily represent the official views of Harvard Catalyst, Harvard University and its affiliated academic healthcare centres or the National Institutes of Health.

Conflict of interest: None declared.

Copyright (OERS 2014 


\section{Introduction}

Precapillary pulmonary hypertension is defined haemodynamically by right heart catheterisation as a mean pulmonary artery pressure $(\mathrm{mPAP}) \geqslant 25 \mathrm{mmHg}$ and pulmonary artery wedge pressure (PAWP) $\leqslant 15 \mathrm{mmHg}$ [1]. This definition applies to a heterogeneous group of disorders associated with abnormalities in the pulmonary vasculature that restrict blood flow and can lead to right heart failure. World Health Organization (WHO) group 1 pulmonary arterial hypertension (PAH) describes those in whom pulmonary hypertension is not due to left heart disease (group 2), respiratory disease (group 3 ) or chronic pulmonary emboli (group 4), but is idiopathic or resulting from genetic predisposition, congenital heart disease, connective tissue disease, portal hypertension or drugs/toxins, among other causes [2]. Distinguishing precapillary pulmonary hypertension (groups 1, 3 and 4) from post-capillary or pulmonary venous hypertension is critical and requires accurate measurement of PAWP, as many patients present with risk factors for both $\mathrm{PAH}$ and pulmonary venous hypertension.

Appropriate measurement of PAWP requires careful thought about ventilation mode and timing within the respiratory cycle. Variation across respiratory phases can be profound and create measurement dilemmas and interobserver variability [3]. At the time of initial use of the Cournand catheter in the 1940s, right heart pressures were recorded as averages throughout the respiratory cycle $[4,5]$; this approach was continued into the 1950s and 1960s [6, 7]. Conversely, current consensus statements recommend measurement of PAWP at the end of exhalation [8]. This recommendation, however, is not universally applied. In many catheterisation labs and intensive care units, a computer software-determined mean across multiple respiratory cycles is reported instead [9-11]. In spontaneously breathing patients, this leads to documentation of a PAWP that is lower than had it been measured manually at end-exhalation [12]. This practice could lead to misclassification of individuals with pulmonary venous hypertension as having $\mathrm{PAH}$, though it is uncertain whether use of end-expiratory PAWP represents the most physiological approach. Direct measurement of left ventricular end-diastolic pressure (LVEDP) has been proposed to provide a more accurate assessment [13]. However, the effects of respiratory-related intrathoracic pressure shifts on LVEDP have not been previously well described.

We undertook this study to examine the performance of mean versus end-expiratory PAWP in the evaluation of precapillary pulmonary hypertension, using clinical characterisation as the comparison standard.

\section{Methods}

The study was approved by the Partners Human Research Committees, the institutional review board at Massachusetts General Hospital (Boston, MA, USA). All included patients had undergone right heart catheterisation at Massachusetts General Hospital between March 1, 2010 and December 31, 2011, with findings of precapillary pulmonary hypertension ( $\mathrm{mPAP} \geqslant 25 \mathrm{mmHg}$ and PAWP $\leqslant 15 \mathrm{mmHg}$ ) on the finalised catheterisation report. Our experience in this laboratory has been the reporting of haemodynamic values as means across the respiratory cycle, thus our criteria was chosen to allow for identification of patients with "controversial" PAWP; $\leqslant 15 \mathrm{mmHg}$ as a respiratory mean but $>15 \mathrm{mmHg}$ at end exhalation. Of those undergoing multiple catheterisations only a single catheterisation was included, which was chosen if it was performed with vasoreactivity testing or, if not, if it was the most recent. Patients receiving invasive or noninvasive positive pressure ventilation or continuous positive airway pressure (CPAP) during catheterisation were excluded.

\section{Haemodynamic measurements}

Catheterisation tracings were individually reviewed to verify that MPAP and PAWP as a mean across the respiratory cycle (PAWPmrc) were appropriate for inclusion. From this, PAWPmrc and an end-expiratory PAWP were recorded independently of the documented PAWP for the study. For inclusion, PAWPmrc had to be $\leqslant 15 \mathrm{mmHg}$. Studies in which haemodynamic tracings were not evident were excluded. From the catheterisation report, right atrial pressure (RAP), cardiac output and pulmonary vascular resistance (PVR) were recorded. All haemodynamic measurements were made prior to clinical characterisation of included patients. The zero reference level for the catheterisation laboratory was the mid-axillary line.

If performed, LVEDP was recorded from the catheterisation report. For studies in which left ventricular tracings were available on an appropriate scale $(0-40 \mathrm{mmHg})$, tracings were similarly reviewed with independent recording of LVEDP at end expiration and as a respiratory cycle mean (LVEDPmrc); defined as the average of the highest and lowest LVEDP within the saved tracing.

\section{Clinical characterisation}

Each subject's catheterisation report and medical record were reviewed to identify age, sex, body mass index (BMI) and relevant cardiopulmonary history. From medical notes and summaries, any documentation of 
clinical history of the following conditions was noted: WHO group 1 causes of PAH; congestive heart failure (CHF); obstructive lung disease (not including asthma); restrictive lung disease; chronic thromboembolic pulmonary hypertension (CTEPH); obstructive sleep apnoea (OSA); diabetes mellitus; coronary artery disease; and hypertension. Patients with PAH could be newly diagnosed or already on treatment at the time of catheterisation. Current echocardiography findings, if performed within 1 year prior to or 1 month following catheterisation, were recorded to include evidence of systolic dysfunction, diastolic dysfunction (left ventricular hypertrophy or Doppler evidence of impaired relaxation), aortic or mitral valvular disease (regurgitation, stenosis or replacement) and right ventricular abnormalities (hypertrophy, hypokinesis or dilation). Left atrial size was reported, with left atrial enlargement defined as a diameter $>40 \mathrm{~mm}$.

Clinical and echocardiographic data were used to classify patients as having a precapillary (isolated WHO group $1 \mathrm{PAH}, \mathrm{CTEPH}$, obstructive lung disease or restrictive lung disease), post-capillary (isolated clinical diagnosis of $\mathrm{CHF}$ or systolic, diastolic, or valvular dysfunction on echocardiography), or mixed phenotype. Those with no specific precapillary or post-capillary phenotype were grouped separately. Specific phenotypic classification criteria are shown in table 1.

\section{Statistical analysis}

For all subjects, agreement between reported PAWP, end-expiratory PAWP and PAWPmrc was assessed to comment on reporting practices in our institution. Using ANOVA, Wilcoxon rank-sum and the Chisquared test, clinical and haemodynamic parameters were compared between precapillary, post-capillary and mixed phenotype groups to comment on our algorithm's agreement with haemodynamic findings. Among those with the precapillary phenotype, the prevalence of end-expiratory PAWP $>15 \mathrm{mmHg}$ was calculated. The precapillary group was divided between those with end-expiratory PAWP $>15 \mathrm{mmHg}$ versus $\leqslant 15 \mathrm{mmHg}$. Haemodynamic and clinical characteristics were compared between groups using the t-test, Wilcoxon rank-sum test or Chi-squared testing as appropriate. A p-value of 0.05 was used to assess for significance.

For all subjects, variables associated with magnitude of PAWP respiratory variation were assessed using the t-test or Pearson correlation coefficient. Multivariable linear regression modelling was used to estimate the association between PAWP respiratory variation and covariates including age, sex, BMI, obstructive lung disease, restrictive lung disease, CHF and OSA. For catheterisations associated with interpretable left ventricular tracings, the magnitude of LVEDP respiratory variation was also correlated with PAWP respiratory variation, and differences in respiratory variation between each LVEDP and PAWP pair were described by Bland-Altman analysis. A statistical software package (SAS version 9.3; SAS Institute Inc., Cary, NC, USA) was used for all analyses.

\section{Results}

Initial criteria identified 462 catheterisations involving 410 patients. 30 catheterisations were excluded due to positive pressure ventilation or CPAP and 33 due to inability to view tracings. 16 catheterisations were excluded due to PAWP tracings demonstrating elevated PAWPmrc and six catheterisations due to normal

TABLE 1 Definition of the phenotypes

Precapillary phenotype

Post-capillary phenotype

Mixed phenotype
Diagnosis of a precapillary disease ${ }^{\#}$ AND

No clinical diagnosis of CHF AND

No moderate/severe mitral or aortic valve disease or replacement, systolic dysfunction or diastolic dysfunction ${ }^{+}$on echocardiogram

Clinical diagnosis of CHF OR moderate/severe mitral or aortic valve disease or replacement, systolic dysfunction or diastolic dysfunction ${ }^{+}$on echocardiogram AND

No diagnosis of precapillary disease ${ }^{\#}$

Clinical diagnosis of CHF OR moderate/severe mitral or aortic valve disease or replacement, systolic dysfunction ${ }^{\star}$ or diastolic dysfunction ${ }^{+}$on echocardiogram AND Diagnosis of a precapillary disease ${ }^{\#}$

CHF: congestive heart failure. ${ }^{\#}$ : diagnosis of World Health Organization group 1 pulmonary hypertension, chronic thromboembolic pulmonary hypertension, obstructive lung disease or restrictive lung disease; " indicated by reduced ejection fraction $(<50 \%)$ or wall motion abnormalities; ${ }^{+}$: indicated by left ventricular hypertrophy or Doppler evidence of impaired relaxation. 
TABLE 2 Characteristics of precapillary, post-capillary and mixed phenotype groups

\begin{tabular}{|c|c|c|c|c|}
\hline & Precapillary & Post-capillary & Mixed & p-value ${ }^{\#}$ \\
\hline Subjects $n$ & 93 & 134 & 76 & \\
\hline End-expiratory PAWP mmHg & $12.8 \pm 4.0$ & $16.0 \pm 3.1$ & $14.0 \pm 3.4$ & $<0.0001$ \\
\hline PAWPmrc mmHg & $9.4 \pm 3.2$ & $12.5 \pm 2.2$ & $11.0 \pm 2.7$ & $<0.0001$ \\
\hline PVR dyn $\cdot \mathbf{s} \cdot \mathrm{cm}^{-5}$ & $379(280-639)$ & 240 (178-309) & $320(234-541)$ & $<0.0001$ \\
\hline Left atrial diameter $>40 \mathrm{~mm}^{\pi}$ & 5.8 & 62.1 & 43.7 & $<0.0001$ \\
\hline
\end{tabular}

Data are presented as mean \pm SD, median (interquartile range) or \%, unless otherwise stated. PAWP: pulmonary artery wedge pressure; PAWPmrc: PAWP as mean across the respiratory cycle; PVR: pulmonary vascular resistance. \#: comparisons between all three groups; for pairwise comparisons there was no difference in PVR between precapillary and mixed patients, for all other pairwise comparisons $p<0.05 ; "$ : results only for those with echocardiogram data (precapillary: $n=52$; post-capillary: $n=124$; mixed: $n=71$ ).

mPAP on tracings. 48 additional catheterisations were excluded as they were multiple catheterisations involving a single patient. After the detailed exclusions, 329 catheterisations were included in the study.

Reported PAWP for the entire population was mean \pm SD $11.6 \pm 2.9 \mathrm{mmHg}$. Reported PAWP was similar to measured PAWPmrc $(11.3 \pm 2.9)$ but significantly lower than end-expiratory PAWP (14.6 \pm 3.7$)$. Reported PAWP and PAWPmrc agreed in $50 \%$ of cases and were within $\pm 1 \mathrm{mmHg} 80 \%$ of the time. In comparison, reported PAWP and end-expiratory PAWP agreed $9 \%$ of the time and were within $\pm 1 \mathrm{mmHg} 28 \%$ of the time. Of the entire population, 141 (42.9\%) had end-expiratory PAWP $>15 \mathrm{mmHg}$.

\section{Clinical phenotypes}

$93(28.3 \%)$ subjects met the criteria for the precapillary pulmonary hypertension phenotype, $134(40.7 \%)$ for the post-capillary phenotype, $76(23.1 \%)$ for the mixed phenotype, and 26 did not meet criteria for either pre- or post-capillary pulmonary hypertension. The precapillary pulmonary hypertension group contained subjects with obstructive lung disease $(n=22)$, restrictive lung disease $(n=15)$, WHO group 1 PAH $(n=21)$, CTEPH $(n=16)$, or more than one disease $(n=19$; most commonly obstruction combined with restriction $(n=12))$.

Significant differences in PAWPmrc and end-expiratory PAWP were detected when comparing the precapillary, post-capillary and mixed phenotype groups (table 2); although substantial overlap existed (fig. 1). Consistent with the phenotypes, PVR was greater in precapillary and mixed subjects compared to post-capillary subjects. Left atrial enlargement was uncommon in precapillary subjects, present in only three $(5.8 \%)$ out of the 52 subjects for whom echocardiography data were available.

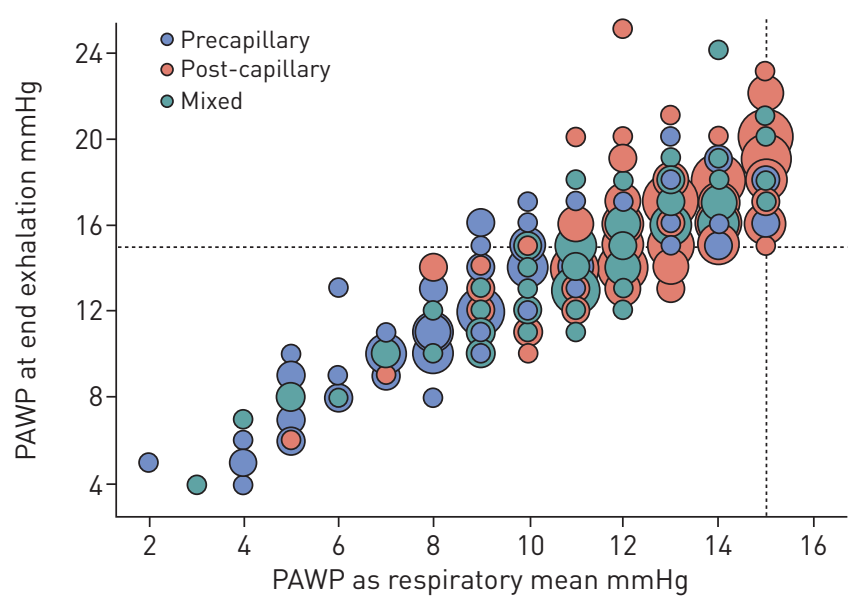

FIGURE 1 Distribution of pulmonary artery wedge pressure (PAWP) at end exhalation versus respiratory mean, as grouped by clinical phenotype. Larger circles represent identical observations in multiple patients. The standard cut-off of $15 \mathrm{mmHg}(\cdots \cdot)$ yields poor phenotype separation, regardless of where PAWP is measured in the respiratory cycle. Blue circles above the standard cut-off represent phenotypically precapillary patients who would have been misclassified as pulmonary venous hypertension using end-expiratory PAWP. 
Precapillary phenotype by end-expiratory PAWP

End-expiratory PAWP >15 mmHg was seen in 27 (29.0\%) phenotypically precapillary subjects. There was no significant difference in age, sex, BMI, type of precapillary disease or comorbid medical conditions between the groups exhibiting end-expiratory PAWP $\leqslant 15 \mathrm{mmHg}$ or $>15 \mathrm{mmHg}$ (table 3). Cardiac output, mPAP, left atrial size and prevalence of echocardiographically abnormal right ventricle were also similar. Those with end-expiratory PAWP $>15 \mathrm{mmHg}$ had significantly lower PVR, greater RAP and greater respiratory variation in PAWP.

\section{WHO group 1 and CTEPH}

56 subjects with WHO group $1 \mathrm{PAH}$ and/or CTEPH (one patient with congenital heart disease and surgically treated CTEPH fit into both groups) were analysed separately. Mean PAWPmrc was $9.0 \pm 3.0 \mathrm{mmHg}$, and end-expiratory PAWP was $12.1 \pm 3.9 \mathrm{mmHg} .13$ (23.2\%) subjects had end-expiratory PAWP $>15 \mathrm{mmHg}$. BMI was greater in the subgroup with end-expiratory PAWP $>15 \mathrm{mmHg}$; otherwise, there were no significant differences in demographics, comorbid conditions or echocardiography data (table 4). Again, significantly greater RAP and more respiratory variation in PAWP were reported in the group with end-expiratory PAWP $>15 \mathrm{mmHg}$.

\section{Respiratory variation in PAWP}

For the full population, the mean difference between end-expiratory PAWP and PAWPmrc (PAWPee-mrc) was $3.3 \pm 1.9 \mathrm{mmHg}$, ranging to $13 \mathrm{mmHg}$ (fig. $2 \mathrm{a}$ and c). Significant positive correlation was found between PAWPee-mrc and BMI $(\mathrm{r}=0.356, \mathrm{p}<0.0001)$. On univariate analysis, there was no significant relationship between PAWPee-mrc and diagnoses of obstructive or restrictive lung disease or CHF. OSA was associated with greater PAWPee-mrc and increased age with lower PAWPee-mrc. On multivariate analysis, a significant relationship was observed between PAWPee-mrc and BMI $(\mathrm{p}<0.0001)$, while there was a trend towards increased PAWPee-mrc in those with obstructive lung disease $(\mathrm{p}=0.0548)$.

TABLE 3 Characteristics of precapillary phenotype patients according to end-expiratory pulmonary artery wedge pressure (PAWP)

End-expiratory PAWP

p-value

\begin{tabular}{ccc}
\hline$\leqslant 15 \mathbf{~ m m H g}$ & $>15 \mathbf{~ m m H g}$ & \\
66 & 27 & \\
$59.3 \pm 14.8$ & $59.4 \pm 14.1$ & 0.98 \\
54.6 & 59.3 & 0.68 \\
$26.9(23.5-32.2)$ & $29.4(25.2-33.3)$ & 0.20 \\
$34.5(28-47)$ & $33(27-47)$ & 0.74 \\
$422(298-658)$ & $296(203-630)$ & 0.033 \\
$4.67(3.70-5.97)$ & $5.33(4.15-6.27)$ & 0.40 \\
$5(4-7)$ & $8(7-12)$ & $<0.0001$ \\
$11(9-13)$ & $17(16-18)$ & $<0.0001$ \\
$8(6-10)$ & $13(12-14)$ & 0.0001 \\
$3(2-4)$ & $5(3-6)$ & 0.27 \\
68.6 & 52.9 & 0.32 \\
$33(30-37)$ & $34(30-39)$ & 0.75 \\
$27(40.9)$ & $12(44.4)$ & 0.48 \\
$22(33.3)$ & $7(25.9)$ & 0.43 \\
$20(30.3)$ & $6(22.2)$ & 0.48 \\
$14(21.2)$ & $4(14.8)$ & 0.42 \\
$8(12.1)$ & $5(18.5)$ & 0.71 \\
$29(43.9)$ & $13(48.2)$ & 0.65 \\
$12(18.2)$ & $6(22.2)$ & 0.070 \\
$9(13.6)$ & $8(29.6)$ &
\end{tabular}

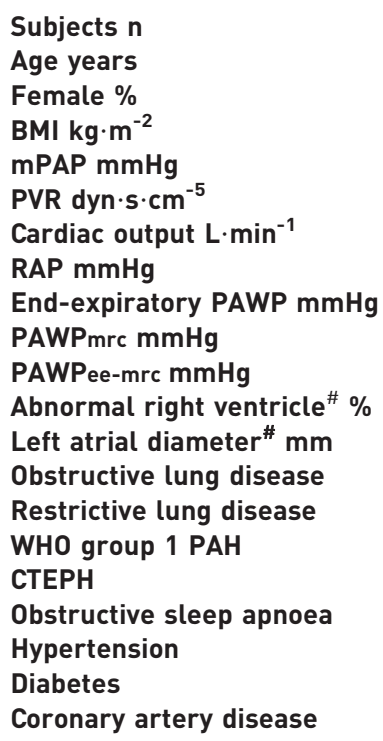

Data are presented as mean $\pm S D$, median (interquartile range) or $n(\%)$, unless otherwise stated. BMI: body mass index; mPAP: mean pulmonary artery pressure; PVR: pulmonary vascular resistance; RAP: right atrial pressure; PAWPmrc: PAWP as mean across respiratory cycle; PAWPee-mrc: mean difference between end-expiratory PAWP and PAWPmrc; WHO: World Health Organization; PAH: pulmonary arterial hypertension; CTEPH: chronic thromboembolic pulmonary hypertension. * : results only for those with echocardiogram data $(n=35$ and $n=17$ for end-expiratory PAWP $\leqslant 15$ and $>15 \mathrm{mmHg}$, respectively). 
TABLE 4 Characteristics of World Health Organization group 1 and chronic thromboembolic pulmonary hypertension patients according to end-expiratory pulmonary artery wedge pressure (PAWP)

\begin{tabular}{|c|c|c|c|}
\hline & \multicolumn{2}{|c|}{ End-expiratory PAWP } & \multirow[t]{2}{*}{ p-value } \\
\hline & $\leqslant 15 \mathrm{mmHg}$ & $>15 \mathrm{mmHg}$ & \\
\hline Subjects $\mathrm{n}$ & 43 & 13 & \\
\hline Age years & $56.1 \pm 15.5$ & $58.9 \pm 10.3$ & 0.54 \\
\hline Female \% & 65.1 & 69.2 & 0.78 \\
\hline $\mathrm{BMI} \mathrm{kg} \cdot \mathrm{m}^{-2}$ & $27.4(23.5-30.9)$ & $31.8(27.6-35.9)$ & 0.015 \\
\hline mPAP $\mathrm{mmHg}$ & $41(34-49)$ & $47(39-62)$ & 0.099 \\
\hline PVR dyn $\cdot 5 \cdot \mathrm{cm}^{-5}$ & $520(361-846)$ & $706(396-1089)$ & 0.36 \\
\hline Cardiac output L. $\min ^{-1}$ & $4.84(4.05-6.55)$ & $4.85(4.15-5.40)$ & 0.69 \\
\hline RAP $\mathrm{mmHg}$ & $6(5-9)$ & $10(8-12)$ & 0.0028 \\
\hline End-expiratory PAWP $\mathrm{mmHg}$ & $11(9-12)$ & $17(16-18)$ & $<0.0001$ \\
\hline PAWPmrc mmHg & $8(6-9)$ & $13(12-15)$ & $<0.0001$ \\
\hline PAWPee-mrc mmHg & $3(2-3)$ & $5(4-6)$ & 0.0007 \\
\hline Abnormal right ventricle ${ }^{\#} \%$ & 70.8 & 77.8 & 0.69 \\
\hline Left atrial diameter ${ }^{\#} \mathrm{~mm}$ & $33(30-37)$ & $38(34-39)$ & 0.097 \\
\hline Obstructive sleep apnoea & $5(11.6)$ & $4(30.8)$ & 0.10 \\
\hline Hypertension & $14(32.6)$ & $5(38.5)$ & 0.69 \\
\hline Diabetes & $5(11.6)$ & $3(23.1)$ & 0.30 \\
\hline Coronary artery disease & $5(11.6)$ & $3(23.1)$ & 0.30 \\
\hline
\end{tabular}

Data are presented as mean $\pm S D$, median (interquartile range) or $n(\%)$, unless otherwise stated. BMI: body mass index; mPAP: mean pulmonary artery pressure; PVR: pulmonary vascular resistance; RAP: right atrial pressure; PAWPmrc: PAWP as mean across respiratory cycle; PAWPee-mrc: mean difference between end-expiratory PAWP and PAWPmrc. ${ }^{*}$ : results only for those with echocardiogram data $(n=24$ and $n=9$ for endexpiratory PAWP $\leqslant 15$ and $>15 \mathrm{mmHg}$, respectively).

\section{Respiratory variation in LVEDP}

$75(22.8 \%)$ patients had assessment of LVEDP at the time of right heart catheterisation; of these, 30 catheterisations saved tracings at an appropriate scale for evaluation of LVEDP. The mean difference between end-expiratory LVEDP and LVEDPmrc (LVEDPee-mrc) was $4.1 \pm 3.0 \mathrm{mmHg}$, ranging to $16 \mathrm{mmHg}$ (fig. $2 \mathrm{~b}$ and c). There was a very strong correlation between PAWPee-mrc and LVEDPee-mrc $(\mathrm{r}=0.859$, $\mathrm{p}<0.0001)$. Respiratory variation in LVEDP was, on average, $0.5 \mathrm{mmHg}$ (95\% CI $-0.1-1.1)$ greater than PAWPee-mrc (fig. 3).

\section{Discussion}

In this study, end-expiratory PAWP had poor sensitivity for identifying patients with precapillary pulmonary hypertension, as defined by clinical history. In an attempt to classify patients as having precapillary pulmonary hypertension using more than just PAWP, we defined clinical phenotypes that were then compared with PAWP, both as a respiratory cycle mean and at end expiration. Among patients with precapillary disease who had no evidence, clinically or by echocardiogram, of left heart dysfunction, elevation in end-expiration PAWP was common (29\%). Strict use of end-expiratory PAWP would have led to misclassification of this group as having pulmonary venous hypertension. These patients did not appear to be different to comparable patients with strictly normal end-expiratory PAWP, with similarities in demographics, medical history, echocardiogram findings and most catheterisation haemodynamics. However, right atrial pressure was significantly higher in the group with elevated end-expiratory PAWP, and there was also greater respirophasic variation in PAWP in these patients.

It is likely that the mechanism for "false" elevation in end-expiratory PAWP seen in our cohort relates to intrathoracic pressure swings throughout the respiratory cycle. During catheterisation, use of endexpiratory PAWP assumes that intrathoracic pressure is approximately equal to atmospheric pressure at end-exhalation and, thus, measured PAWP reflects a transmural left ventricular filling pressure. In the setting of mechanical ventilation [14, 15], obesity [16], or chronic obstructive pulmonary disease (COPD) [17-21], end-expiratory intrathoracic pressure can be significantly greater than atmospheric pressure. In these scenarios, use of end-expiratory PAWP overestimates true transmural PAWP (as well as RAP). BOERRIGTER et al. [20] recently reached similar conclusions in exercising COPD patients. PAWP averaged over the respiratory cycle provided a more accurate estimate of transmural PAWP, as determined using simultaneous oesophageal pressure measurements [20]. 
a)
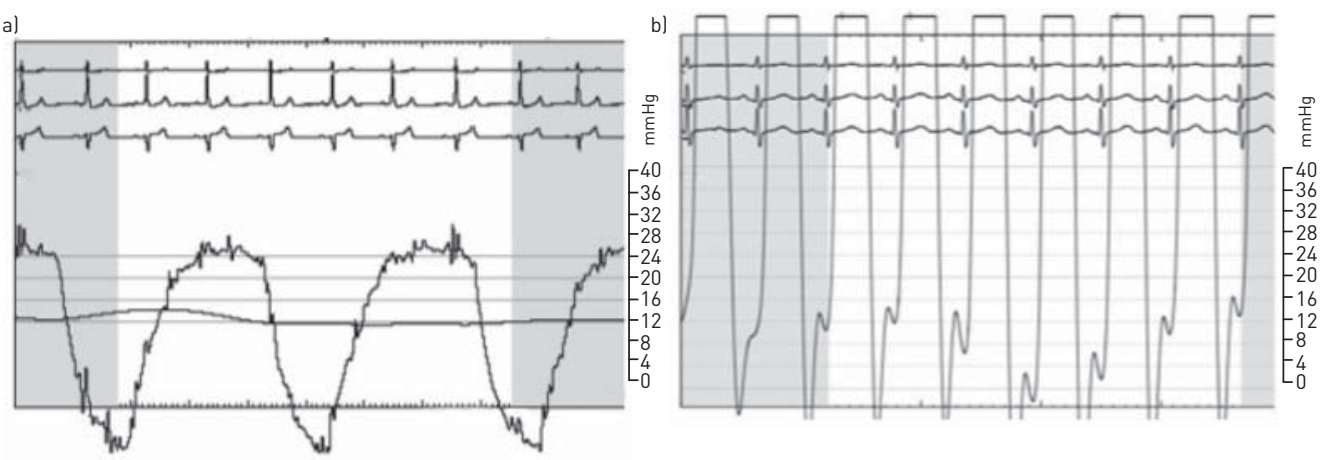

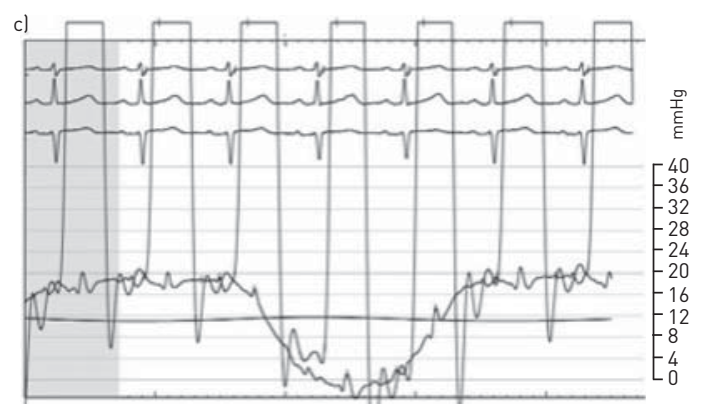

FIGURE 2 a) Pulmonary artery wedge pressure tracing from one subject demonstrating extreme respirophasic variation in wedge pressure, with $>10 \mathrm{mmHg}$ difference between end-expiratory and respiratory mean measurements. b) Left ventricular pressure tracing from a second subject demonstrating large respirophasic variation in left ventricular enddiastolic pressure. c) Simultaneous wedge pressure and left ventricular pressure tracings from a third subject, illustrating overlapping respirophasic variation.

In many of our subjects with elevated end-expiratory PAWP and RAP, we hypothesised that these high pressures reflect elevated end-expiratory intrathoracic pressure with normal transmural right and left atrial pressures. As additional support of this, we noted that those with elevated end-expiratory PAWP demonstrated significantly more respiratory variation in PAWP than those with lower end-expiratory PAWP. Interpretation of this finding is limited by selection bias, i.e. a subject with end-expiratory PAWP of $20 \mathrm{mmHg}$ must have a PAWPee-mrc difference of at least $5 \mathrm{mmHg}$ to be included. However, this larger respiratory variation may be a surrogate for positive end-expiratory intrathoracic pressure, which must be overcome before establishment of inspiratory airflow.

We further analysed the distribution of respirophasic wedge pressure variation within a diverse population of subjects undergoing catheterisation. While normal respiratory cycle-ventricular filling interactions are complex [22, 23], the most significant factor influencing respirophasic wedge pressure variation appears to be intrathoracic pressure variation. Previous work in COPD has supported a close relationship between variable intrathoracic pressure and measured wedge pressures [19-21, 24, 25]. For example, RiCE et al. [19] demonstrated very tight correlation (slope 1.04, $\mathrm{r}=0.98$ ) between changes in oesophageal and wedge pressures during respiration in COPD patients. One would anticipate COPD and other conditions associated with spontaneous positive end-expiratory intrathoracic pressure show greater PAWPee-mrc. In our study, a nonsignificant trend towards increased PAWPee-mrc in those with obstructive disease was reported; the study was probably underpowered to detect a significant difference in this group.

The magnitude of phasic PAWP change was strongly correlated with BMI. Obesity has been repeatedly associated with positive end-expiratory intrathoracic pressure during spontaneous breathing, particularly in the supine position $[16,26,27]$. Among our WHO group 1 PAH and/or CTEPH subjects, those in the high end-expiratory PAWP group had higher BMI. Similarly, recent analysis of the REVEAL (Registry to Evaluate Early and Long-term Pulmonary Arterial Hypertension Disease Management) registry found higher prevalence of obesity in those with higher PAWP [28].

As positive end-expiratory intrathoracic pressure impacts all catheterisation pressure measurements, those with higher end-expiratory PAWP should also have higher measured (though not transmural) mPAP. Interestingly, in the REVEAL study mPAP and RAP were both greater in patients with higher levels of PAWP [28]. In our analysis of WHO group 1 and CTEPH patients, a trend towards increased mPAP was seen in the group with end-expiratory PAWP $>15 \mathrm{mmHg}$. It should be noted that mPAP measurements 


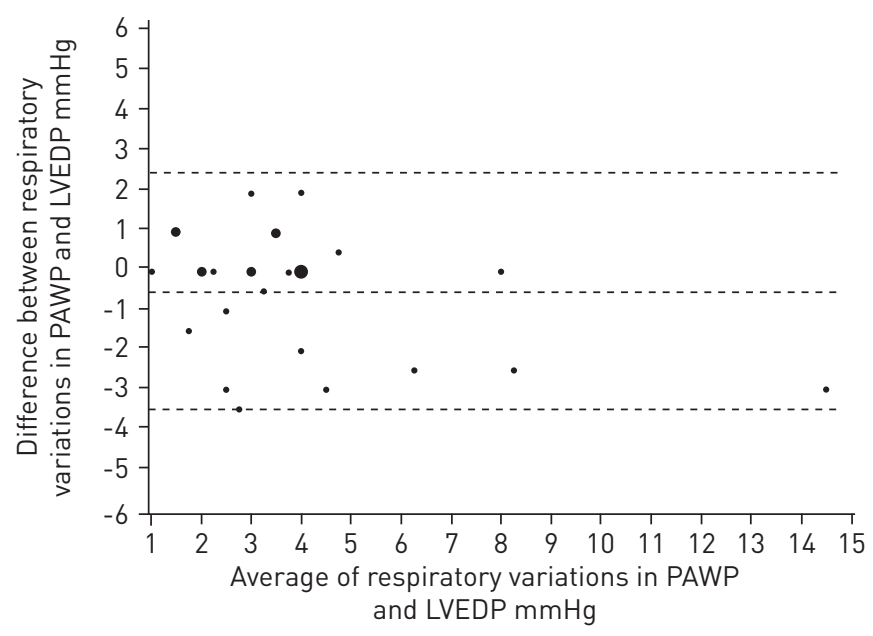

FIGURE 3 Bland-Altman plot of difference between PAWPee-mrc and LVEDPee-mrc versus average of respiratory variation in PAWPee-mrc and LVEDPee-mrc. A positive value on the $y$-axis indicates subjects for whom respiratory variation in pulmonary artery wedge pressure was greater than respiratory variation in left ventricular end-diastolic pressure. Larger circles represent identical observations in multiple patients. Mean difference: $-0.5 \mathrm{mmHg}$ ( $95 \% \mathrm{CI}-1.1-0.1)$; limits of agreement: -3.5-2.5. PAWPee-mrc: mean difference between end-expiratory pulmonary artery wedge pressure (PAWP) and PAWP as respiratory mean; LVEDPee-mrc: mean difference between left ventricular end-diastolic pressure (LVEDP) and LVEDP as respiratory mean.

were obtained from catheterisation reports, and thus were not necessarily end-expiratory values. If our hypothesis holds true we would anticipate end-expiratory mPAP to be even greater in those with endexpiratory PAWP $>15 \mathrm{mmHg}$, further exaggerating this difference between groups.

Although reflective of a small sample $(n=30)$ of subjects, we additionally demonstrated respirophasic change in LVEDP, which correlated well with respirophasic variability in PAWP. The relationship between absolute values of LVEDP and PAWP has been previously described, with closer correlations found in studies in which the respiratory phase of PAWP and LVEDP is specifically observed and uniformly recorded $[10,29]$, compared to studies in which tracings were not individually reviewed and/or were not necessarily measured in the same phase of respiration $[13,30]$. Routine measurement of LVEDP, in addition to adding procedural risk, is subject to the same debates regarding respiratory variability as PAWP.

Clinical decisions made using data obtained by nonstandardised methodology can have substantial consequence. As recently analysed by KovACS et al. [31], the choice of zero reference level has very clinically significant effects on interpretation of PAWP, altering the diagnosis in up to $31 \%$ of patients. Similarly, the practical question of where, within the respiratory cycle, to measure PAWP carries great importance and is worthy of further discussion and investigation. Strict use of PAWPmrc can underdiagnose pulmonary venous hypertension; we also argue that use of end-expiratory PAWP underdiagnoses PAH. Inaccurate interpretation of PAWP has the potential to lead to inappropriate use of PAH therapies (both overuse and underuse). While published inclusion criteria for most randomised clinical trials in PAH specified PAWP $<15 \mathrm{mmHg}$ or $\leqslant 15 \mathrm{mmHg}$, none specified where in the respiratory cycle this value was measured [32-40]. Interestingly, in major PAH registries and trials that report baseline PAWP, means ranged between $7.5 \mathrm{mmHg}$ and $10 \mathrm{mmHg}[32,35,37-45]$, matching more closely with our WHO group 1 PAWPmrc $(9.1 \pm 3.3)$ in comparison to end-expiratory PAWP $(12.3 \pm 4.2)$. We hypothesise that many or all of these studies utilised PAWPmrc and, had end-expiratory PAWP been used instead, some of these subjects would have been, possibly inappropriately, excluded from the trials.

The limitations of this study are important to discuss. Our study occurred at a single centre, thus, wedge pressure reporting practices at our institution may not mirror other centres. Our methods of designating phenotypes, while similar to those commonly used in practice, were not specifically validated. Phenotype designation required chart documentation of pertinent diagnoses; as these were not universally performed, radiological and pulmonary function studies were not routinely reviewed for verification. While our total study population was large $(n=329)$, our phenotyping strategies decreased evaluated numbers to smaller groups. Our power to detect meaningful differences between precapillary subjects with high versus low endexpiratory PAWP, for example, may be insufficient. The REVEAL analysis detected differences in several medical comorbidities between those with high and low PAWP [28]. We were probably underpowered to detect such differences. 
We conclude that patients with precapillary pulmonary hypertension may be mislabelled as having pulmonary venous hypertension if end-expiratory wedge pressure is always utilised, especially in the setting of obesity or obstructive lung disease. The addition of clinical phenotyping can enhance interpretation of these often complex haemodynamic measurements.

\section{Acknowledgements}

Statistical support was provided by Harvard Catalyst (Harvard Clinical and Translational Science Center, Boston, MA, USA).

\section{References}

1 Galie N, Hoeper MM, Humbert M, et al. Guidelines for the diagnosis and treatment of pulmonary hypertension: the Task Force for the Diagnosis and Treatment of Pulmonary Hypertension of the European Society of Cardiology (ESC) and the European Respiratory Society (ERS), endorsed by the International Society of Heart and Lung Transplantation (ISHLT). Eur Heart J 2009; 30: 2493-2537.

2 Simonneau G, Robbins IM, Beghetti M, et al. Updated clinical classification of pulmonary hypertension. J Am Coll Cardiol 2009; 54: Suppl. 1, S43-S54.

3 Al-Kharrat T, Zarich S, Amoateng-Adjepong Y, et al. Analysis of observer variability in measurement of pulmonary artery occlusion pressures. Am J Respir Crit Care Med 1999; 160: 415-420.

4 Bloomfield RA, Lauson HD, Cournand A, et al. Recording of right heart pressures in normal subjects and in patients with chronic pulmonary disease and various types of cardio-circulatory disease. J Clin Invest 1946; 25: 639-664.

5 Cournand A, Motley HL, Werko L, et al. Physiological studies of the effects of intermittent positive pressure breathing on cardiac output in man. Am J Physiol 1948; 152: 162-174.

6 Segel N, Hudson WA, Harris P, et al. The circulatory effects of electrically induced changes in ventricular rate at rest and during exercise in complete heart block. J Clin Invest 1964; 43: 1541-1550.

Harris P. Influence of acetylcholine on the pulmonary arterial pressure. Br Heart J 1957; 19: 272-278.

8 McLaughlin VV, Archer SL, Badesch DB, et al. ACCF/AHA 2009 Expert Consensus Document on Pulmonary HypertensionA Report of the American College of Cardiology Foundation Task Force on Expert Consensus Documents and the American Heart Association Developed in Collaboration With the American College of Chest Physicians; American Thoracic Society, Inc.; and the Pulmonary Hypertension Association. J Am Coll Cardiol 2009; 53: 1573-1619.

9 Ahrens TS, Schallom L. Comparison of pulmonary artery and central venous pressure waveform measurements via digital and graphic measurement methods. Heart Lung 2001; 30: 26-38.

10 Ryan JJ, Rich JD, Thiruvoipati T, et al. Current practice for determining pulmonary capillary wedge pressure predisposes to serious errors in the classification of patients with pulmonary hypertension. Am Heart J 2012; 163: 589-594.

11 Maran AG. Variables in pulmonary capillary wedge pressure: variation with intrathoracic pressure, graphic and digital recorders. Crit Care Med 1980; 8: 102-105.

12 Cengiz M, Crapo RO, Gardner RM. The effect of ventilation on the accuracy of pulmonary artery and wedge pressure measurements. Crit Care Med 1983; 11: 502-507.

13 Halpern SD, Taichman DB. Misclassification of pulmonary hypertension due to reliance on pulmonary capillary wedge pressure rather than left ventricular end-diastolic pressure. Chest 2009; 136: 37-43.

14 Hoyt JD, Leatherman JW. Interpretation of the pulmonary artery occlusion pressure in mechanically ventilated patients with large respiratory excursions in intrathoracic pressure. Intensive Care Med 1997; 23: 1125-1131.

15 Schuster DP, Seeman MD. Temporary muscle paralysis for accurate measurement of pulmonary artery occlusion pressure. Chest 1983; 84: 593-597.

16 Behazin N, Jones SB, Cohen RI, et al. Respiratory restriction and elevated pleural and esophageal pressures in morbid obesity. J Appl Physiol 2010; 108: 212-218.

17 Boerrigter B, Trip P, Bogaard HJ, et al. Right atrial pressure affects the interaction between lung mechanics and right ventricular function in spontaneously breathing COPD patients. PLoS One 2012; 7: e30208.

18 Butler J, Schrijen F, Henriquez A, et al. Cause of the raised wedge pressure on exercise in chronic obstructive pulmonary disease. Am Rev Respir Dis 1988; 138: 350-354.

19 Rice DL, Awe RJ, Gaasch WH, et al. Wedge pressure measurement in obstructive pulmonary disease. Chest 1974; 66: 628-632.

20 Boerrigter BG, Waxman AB, Westerhof N, et al. Measuring central pulmonary pressures during exercise in COPD: how to cope with respiratory effects. Eur Respir J 2014; 43: 1316-1325.

21 Naeije R, Boerrigter BG. Pulmonary hypertension at exercise in COPD: does it matter? Eur Respir J2013; 41: 1002-1004.

22 Pinsky MR. Cardiovascular issues in respiratory care. Chest 2005; 128: Suppl. 5, 592S-597S.

23 Lauson HD, Bloomfield RA, Cournand A. The influence of the respiration on the circulation in man; with special reference to pressures in the right auricle, right ventricle, femoral artery and peripheral veins. Am J Med 1946; 1: 315-336.

24 Bahler RC, Chester EH, Belman MJ, et al. Multidisciplinary treatment of chronic pulmonary insufficiency. 4. The influence of intrathoracic pressure variations on increases in pulmonary vascular pressure during exercise in patients with chronic obstructive pulmonary disease. Chest 1977; 72: 703-708.

25 Lim TP, Brownlee WE. Pulmonary hemodynamics in obstructive lung disease. Dis Chest 1968; 53: 113-125.

26 Steier J, Jolley CJ, Seymour J, et al. Neural respiratory drive in obesity. Thorax 2009; 64: 719-725.

27 Pankow W, Podszus T, Gutheil T, et al. Expiratory flow limitation and intrinsic positive end-expiratory pressure in obesity. J Appl Physiol 1998; 85: 1236-1243.

28 Frost AE, Farber HW, Barst RJ, et al. Demographics and outcomes of patients diagnosed with pulmonary hypertension with pulmonary capillary wedge pressures 16 to $18 \mathrm{mmHg}$ : insights from the REVEAL Registry. Chest 2013; 143: 185-195. 
Oliveira RK, Ferreira EV, Ramos RP, et al. Usefulness of pulmonary capillary wedge pressure as a correlate of left ventricular filling pressures in pulmonary arterial hypertension. J Heart Lung Transplant 2014; 33: 157-162.

30 Bitar A, Selej M, Bolad I, et al. Poor agreement between pulmonary capillary wedge pressure and left ventricular end-diastolic pressure in a veteran population. PLoS One 2014; 9: e87304.

31 Kovacs G, Avian A, Olschewski A, et al. Zero reference level for right heart catheterisation. Eur Respir J 2013; 42: 1586-1594.

32 Channick RN, Simonneau G, Sitbon O, et al. Effects of the dual endothelin-receptor antagonist bosentan in patients with pulmonary hypertension: a randomised placebo-controlled study. Lancet 2001; 358: 1119-1123.

33 Galie N, Brundage BH, Ghofrani HA, et al. Tadalafil therapy for pulmonary arterial hypertension. Circulation 2009; 119: 2894-2903.

34 Galie N, Ghofrani HA, Torbicki A, et al. Sildenafil citrate therapy for pulmonary arterial hypertension. $N$ Engl J Med 2005; 353: 2148-2157.

35 Jing ZC, Yu ZX, Shen JY, et al. Vardenafil in pulmonary arterial hypertension: a randomized, double-blind, placebo-controlled study. Am J Respir Crit Care Med 2011; 183: 1723-1729.

36 McLaughlin VV, Oudiz RJ, Frost A, et al. Randomized study of adding inhaled iloprost to existing bosentan in pulmonary arterial hypertension. Am J Respir Crit Care Med 2006; 174: 1257-1263.

37 Olschewski H, Simonneau G, Galie N, et al. Inhaled iloprost for severe pulmonary hypertension. N Engl J Med 2002; 347: 322-329.

38 Rubin LJ, Badesch DB, Barst RJ, et al. Bosentan therapy for pulmonary arterial hypertension. $N$ Engl J Med 2002; 346: 896-903.

39 Simonneau G, Barst RJ, Galie N, et al. Continuous subcutaneous infusion of treprostinil, a prostacyclin analogue, in patients with pulmonary arterial hypertension: a double-blind, randomized, placebo-controlled trial. Am J Respir Crit Care Med 2002; 165: 800-804.

40 Zeng WJ, Xiong CM, Zhao L, et al. Atorvastatin in pulmonary arterial hypertension (APATH) study. Eur Respir J 2012; 40: 67-74.

41 Badesch DB, Raskob GE, Elliott CG, et al. Pulmonary arterial hypertension: baseline characteristics from the REVEAL Registry. Chest 2010; 137: 376-387.

42 Humbert M, Sitbon O, Chaouat A, et al. Pulmonary arterial hypertension in France: results from a national registry. Am J Respir Crit Care Med 2006; 173: 1023-1030.

43 Thenappan T, Shah SJ, Rich S, et al. A USA-based registry for pulmonary arterial hypertension: 1982-2006. Eur Respir J 2007; 30: 1103-1110.

44 Barst RJ, Rubin LJ, Long WA, et al. A comparison of continuous intravenous epoprostenol (prostacyclin) with conventional therapy for primary pulmonary hypertension. N Engl J Med 1996; 334: 296-301.

45 Simonneau G, Rubin LJ, Galie N, et al. Addition of sildenafil to long-term intravenous epoprostenol therapy in patients with pulmonary arterial hypertension: a randomized trial. Ann Intern Med 2008; 149: 521-530. 